Supporting Information for

Molecular Architecture of the Blood Brain Barrier Tight Junction Proteins-A Synergistic Computational and In Vitro Approach

Flaviyan Jerome Irudayanathan, ${ }^{\dagger}$ John P. Trasatti, ${ }^{\ddagger}$ Pankaj Karande, ${ }^{\S}$ and Shikha Nangia ${ }^{\dagger^{*}}$

${ }^{\dagger}$ Department of Biomedical and Chemical Engineering, Syracuse University, Syracuse NY 13244, United States

${ }^{\ddagger}$ Department of Chemistry and Chemical Biology, Rensselaer Polytechnic Institute, Troy, New York 12180, United States

${ }^{\S}$ Department of Chemical and Biological Engineering, Rensselaer Polytechnic Institute, Troy, New York 12180, United States

*Corresponding author:snangia@syr.edu 


\section{METHODS}

\section{Homology Modeling}

Multiple monomer models of claudin-5 were built using servers available at:
1) http://www.sbg.bio.ic.ac.uk/phyre2/html/page.cgi?id=index ${ }^{1}$
2) http://zhanglab.ccmb.med.umich.edu/I-TASSER/2
3) http://swissmodel.expasy.org/ ${ }^{3}$
4) http://www.robetta.org/submit.jsp
5) http://opig.stats.ox.ac.uk/webapps/memoir/php/ ${ }^{4}$

These models were built using the crystal structure of mouse claudin-15 (PDB ID: 4P79) as a template ${ }^{5}$. The missing C-terminal domain was modeled using ab initio modeling available at http://zhanglab.ccmb.med.umich.edu/QUARK/ ${ }^{6}$. The models were serially refined and scored based on the structure quality factor (http://services.mbi.ucla.edu/SAVES/) and membrane protein quality (http://www.bioinfo.ifm.liu.se/ProQM/) along with Ramachandran plot assessment (http://mordred.bioc.cam.ac.uk/ rapper/rampage.php). C- $\alpha$ backbone energies were refined at CABS-fold server ${ }^{7}$ (http://biocomp.chem.uw.edu.pl/CABSfold/index.php), which employs a replica exchange simulation of the backbone energy based on the consensus from the above models. Side chain optimizations were performed using YASARA modeling software ${ }^{8}$. Multiple sequence alignment of classic claudins was performed using Clustal Omega (http://www.ebi.ac.uk/Tools/msa/clustalo/) and the alignment was visualized using jalview.

\section{Simulation Hardware}

All simulations were carried out using graphics processor unit (GPU) hardware available at supercomputing facility hosted by XSEDE. Necessary protocols and changes were incorporated in the algorithms to expedite the calculations. GPU hardware remarkably decreased the computation time, typically by $50 \%$, compared to central processing unit (CPUs) hardware. The calculation of $>200 \mu$ s of self-assembly simulations and $>100 \mu$ s of umbrella sampling simulation utilized 200,000 GPU hours of computation time. All timescales reported are actual simulation times and not scaled up for the speed up achieved in MARTINI simulation which is estimated to be a factor of $4^{9,10}$. The total simulation time with scaling up corresponds to $\sim 1.2 \mathrm{~ms}$.

\section{Membrane Insertion and Monomer Simulation}

Hydrophobic thickness and the relative orientation of claudin-5 monomer with respect to lipid bilayer were calculated at the PPM server hosted at http://opm.phar.umich.edu/server.php ${ }^{11}$. Input files for all atom simulations were generated from http://charmmgui.org/?doc=input/membrane ${ }^{12}$. All atom simulations were carried out using NAMD 2.9 molecular dynamics engine ${ }^{13}$ with CHARMM36 force field ${ }^{14,15}$. The system contained 145 POPC molecules, 10930 TIP3 waters and $0.15 \mathrm{M} \mathrm{KCl}$ along with claudin-5 in a simulation box 
of $8 \times 8 \times 10 \mathrm{~nm}^{3}$ size and periodic boundary conditions. The system was energy minimized and serially equilibrated in $N V T$ and $N P T$ ensembles with positional restraints. The production run for $150 \mathrm{~ns}$ was carried out in $N P T$ ensemble without positional restraints at $310.15 \mathrm{~K}$ maintained by Langevin thermostat and $1 \mathrm{~atm}$ maintained by Nosé-Hoover Langevin piston pressure control and 2 fs time steps ${ }^{16,17}$. Long range electrostatic interactions were calculated using particle mesh Ewald algorithm ${ }^{18}$. Cut-off for calculating van der Waal's interactions and electrostatic interactions was set at $1.2 \mathrm{~nm}$ and force-shifted at $1.0 \mathrm{~nm}$. The simulation trajectories were visualized and analyzed using VMD 1.9.2 software and associated plugins (http://www.ks.uiuc.edu/Research/vmd/).

\section{Self-assembly CGMD Simulation}

Equilibrated monomers from the previous steps were assembled in a $4 \times 4$ grid with inter centerof-mass distance of $6 \mathrm{~nm}$ using YASARA model software ${ }^{8}$. Similar strategy was used to generate a larger $8 \times 8$ grid. Two other 16 monomer grids, one with random orientation of claudin-5 and another with inter center-of-mass distance of $8 \mathrm{~nm}$ were also constructed. The monomers in each grid were coarse grained using the MARTINI ${ }^{10,19}$ parameter set for proteins and the secondary structure was maintained using ElNeDyn network ${ }^{20}$. CG lipid bilayers of varying compositions were built around the grids using the Insert membrane (INSANE) script ${ }^{21}$. The box size of each system was adjusted to incorporate the same inter-protein distance between monomers in the periodic image. Typically the membranes were asymmetric by 30-45 lipids in order to accommodate for anisotropies in the monomeric structure. The protein: lipid concentration was $\sim 1: 150$, and the systems were solvated with MARTINI water beads. All systems had a net neutral charge with $0.15 \mathrm{M} \mathrm{NaCl}$.

CGMD simulations were carried out using GROMACS $4.6^{22}$ software suite. The systems were energy minimized using steepest decent algorithm, followed by $40 \mathrm{~ns}$ in $N V T$ ensemble and $1 \mu \mathrm{s}$ in NPT ensemble during which the protein backbone beads had positional restrained. Microsecond long NPT ensemble allowed for proper equilibration of the lipid membrane around the protein. Velocity rescale thermostat ${ }^{23}$ and Parrinello-Rahman barostats ${ }^{24}$ were employed with Verlet buffered pair list cut-off scheme. Dielectric screening constant was set to $\varepsilon_{\mathrm{r}}=15$, which corresponds to the dielectric constant of water $\left(\varepsilon_{\mathrm{r}}=80\right)$ in MARTINI systems ${ }^{10,19}$. The simulations had semi-isotropic pressure coupling in the $x$ and $y$ directions at 1 bar to accommodate for the bilayer equilibration. For the production runs, all positional restraints on the protein were removed and the protein was allowed to equilibrate freely. A $20 \mathrm{fs}$ timestep was used in all simulation runs, and both the energies and coordinates were written at $0.5 \mathrm{~ns}$ frequency.

The C-terminal residues from 191-218 were removed in the 64 monomer system to reduce the box size in the $z$-direction. This modification allowed for the reduction of $\sim 80,000$ beads and enabled faster simulations. Though the intracellular part of the C-terminal starts at 181 , the 
position of 191 was picked based on the observations from the 16 monomer systems where there were no significant interactions observed between residues beyond 190. Experiments have previously shown that the dimerization and strand formation is not affected by $\mathrm{C}$-terminal modification ${ }^{25}$. Further to rule out any discrepancy, we checked this experimental result by running 16 monomer system without $\mathrm{C}$-terminal, and observed no significant change in the dynamics or dimer conformations. However, we did observe a shift in diffusion coefficient from $43.5 \pm 1.6$ to $54.1 \pm 2.310^{-9} \mathrm{~cm}^{2} \mathrm{~s}^{-1}$ which can be accounted for the change in the overall mass. Simulation details and the total simulation times are listed below. The 64 monomer system had 282,972 martini beads corresponding to $\sim 2.5$ million actual atoms, including hydrogens.

In order to rule out orientation bias and/or any configurational bias in dimerization, we repeated self-assembly runs in $4 \times 4$ grid systems with random orientations of claudin- 5 monomers and with inter center-of-mass distance of $8 \mathrm{~nm}$ in DLPC and DPPC bilayers. The dynamics and the dimer orientations sampled remained constant in these simulations. Similarly, some of the simulations were repeated with different random number seeds to confirm the reproducibility of the observed results.

\section{Reverse Mapping}

The coarse grained systems were reverse mapped back to the atomistic system using the protocol described by Wassenaar et al. ${ }^{26}$ The initram.sh script was used for reverse mapping to transform from MARTINI to CHARMM36 force field. Typically, after reverse mapping the system was subjected to energy minimization and short MD simulations of $2 \mathrm{~ns}$ with atomistic topologies and respective all atom simulation parameters described previously. Typically $1 \mu$ s snapshots were reverse mapped and used for analysis. In the case of 64 monomer system only the protein beads were reverse mapped as the system was too large for analysis.

\section{Steered Molecular Dynamics and Umbrella Sampling}

Post reverse mapping, the dimer conformations were analyzed and isolated from the $8 \times 8$ grid system. Using cluster analysis (described below) the centroid average structure of the dimer from the largest self-assembled cluster was extracted and used as input for umbrella sampling simulations. DPPC bilayer was constructed around the dimer as shown in Figure S7 and allowed to equilibrate with positional restraints on backbone beads in NPT ensemble for $1 \mu \mathrm{s}$.

The steered MD was preformed similar to the methodology previously described by Lemkul et al. ${ }^{27}$ Using the pull code implemented in GROMACS a harmonic pulling force at the rate of $0.00005 \mathrm{~nm} / \mathrm{ps}$ was applied on one monomer, keeping the position of the other constant, the pulling force was set at $1000 \mathrm{~kJ} /\left(\mathrm{mol}^{*} \mathrm{~nm}^{2}\right)$. Thirty-two individual configurations were isolated from the pull trajectory, out of which 20 had inter protein distance of $0.1 \mathrm{~nm}$, followed by 12 that had inter protein distance of $0.2 \mathrm{~nm}$, summing up to a total separation distance of $\xi=4.4 \mathrm{~nm}$. The $4.4 \mathrm{~nm}$ separation of inter-protein distance corresponds to $\sim 6.5 \mathrm{~nm}$ of inter COM distance from dimers A-C and E, and for dimer D this would mean an inter COM of $\sim 7.5 \mathrm{~nm}$. These 32 
configurations were then individually simulated using the umbrella sampling simulation for 200 $\mathrm{ns}$, the forces were collected for analysis at $0.02 \mathrm{~ns}$ intervals. Three different force constants values $(750,1000$ and $2000 \mathrm{~kJ} / \mathrm{mol})$ were used in the umbrella sampling of the dimers to arrive at the potential mean of force reported in the main text. The potentials from umbrella sampling windows were unbiased using the weighted histogram analysis (WHAM) method ${ }^{28,29}$.

\section{MDP parameters used in the self-assembly simulations:}

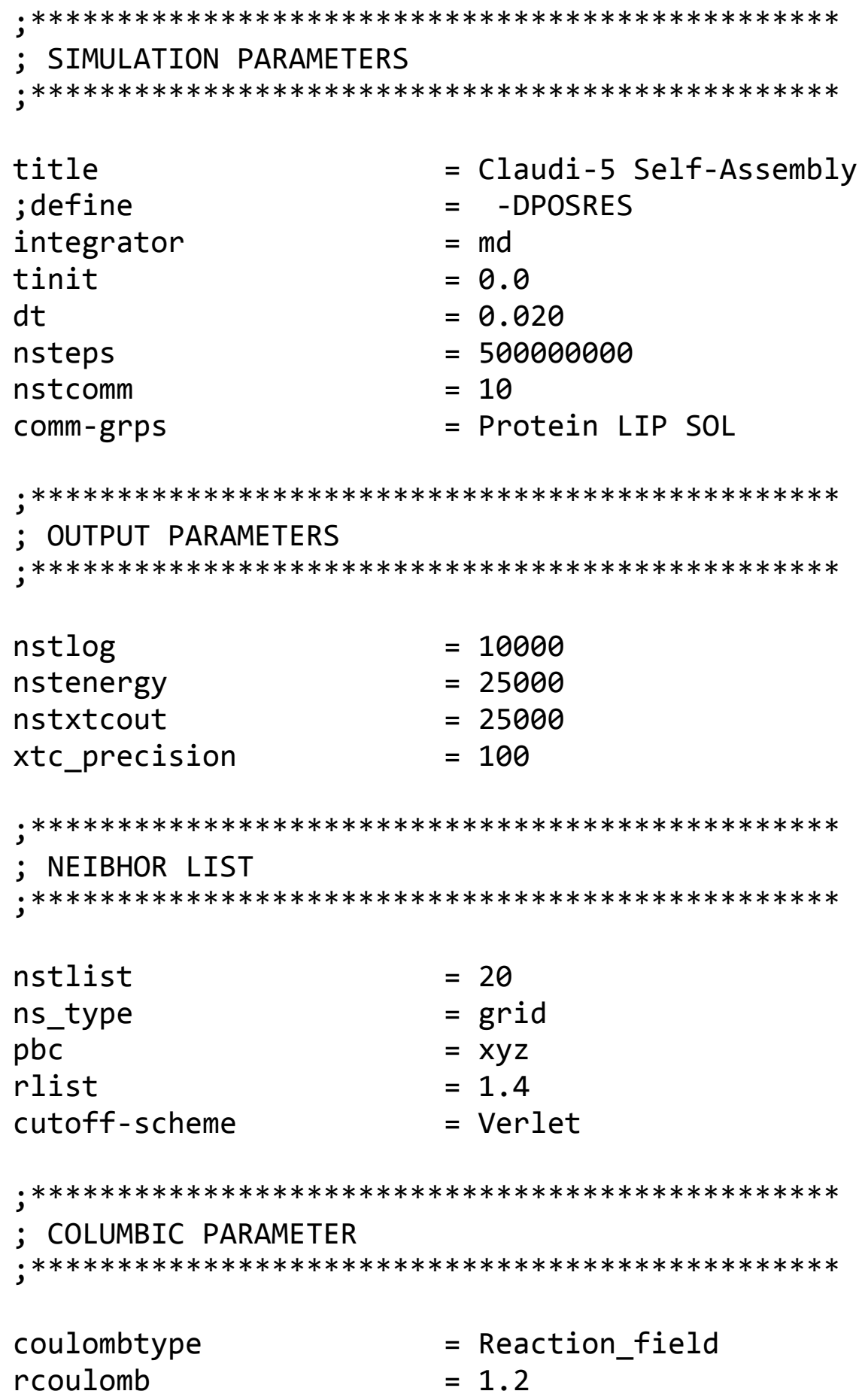




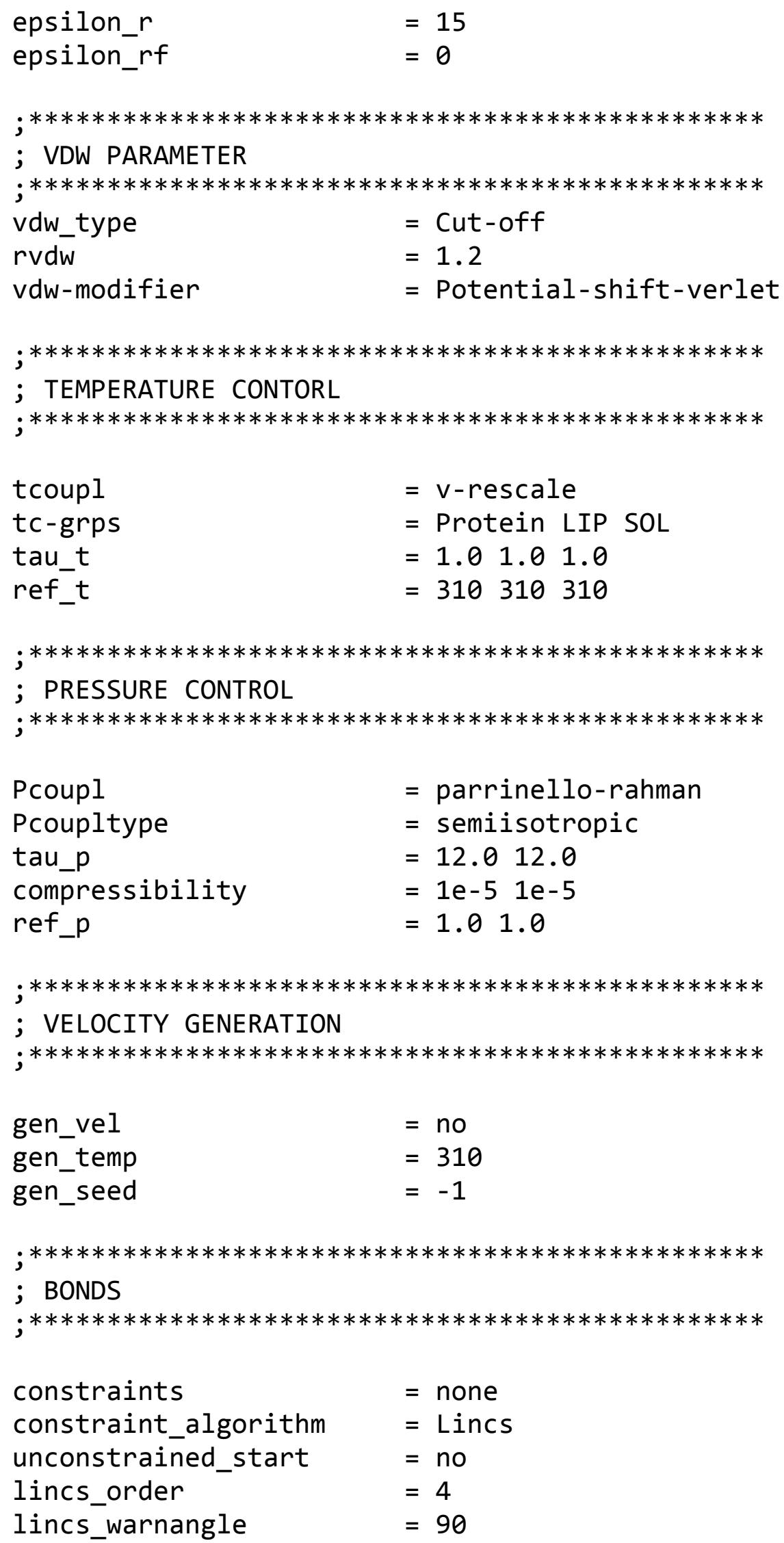


Table S1. List of simulations performed

\begin{tabular}{|c|c|c|c|c|c|}
\hline S.no & System & NPT $(\mu s)$ & Self -assembly $(\mu s)$ & Duplicates & Total $(\mu s)$ \\
\hline \multicolumn{6}{|c|}{ Atomistic } \\
\hline 1 & POPC & 0.02 & 0.15 & - & 0.17 \\
\hline \multicolumn{6}{|c|}{ Coarse Grained } \\
\hline 1 & DLPC & 1 & 10 & 1 & 21 \\
\hline 2 & DYPC & 1 & 10 & - & 11 \\
\hline 3 & DPPC & 1 & 10 & 1 & 21 \\
\hline 4 & POPC & 1 & 10 & 1 & 21 \\
\hline 5 & $\mathrm{PC}$ & 1 & 10 & 1 & 21 \\
\hline 6 & PCS & 1 & 10 & 1 & 21 \\
\hline 7 & $\mathrm{DPC}$ & 1 & 10 & 1 & 21 \\
\hline \multicolumn{6}{|c|}{ Without C-terminal } \\
\hline 1 & DLPC & 1 & 10 & - & 11 \\
\hline 2 & DPPC & 1 & 10 & - & 11 \\
\hline 3 & 64/DPPC & 1 & 10 & - & 11 \\
\hline \multicolumn{6}{|c|}{ Random Orientation } \\
\hline 1 & DLPC & 1 & 10 & - & 11 \\
\hline 2 & DPPC & 1 & 10 & - & 11 \\
\hline \multicolumn{6}{|c|}{ Inter COM distance $=8 \mathrm{~nm}$} \\
\hline 1 & DLPC & 1 & 10 & - & 11 \\
\hline 2 & DPPC & 1 & 10 & - & 11 \\
\hline \multicolumn{5}{|c|}{ Total Self-assembly simulations } & 214 \\
\hline \multicolumn{5}{|c|}{ Umbrella Sampling } & \multirow{4}{*}{101.5} \\
\hline NPT & $: 1 \mu \mathrm{s} \quad \times 5 \mathrm{di}$ & & $=5.0 \mu \mathrm{s}$ & & \\
\hline SMD & $: 0.1 \mu \mathrm{s} \times 5 \mathrm{di}$ & & $=0.5 \mu \mathrm{s}$ & & \\
\hline US & $: 0.2 \mu \mathrm{s} \times 5 \mathrm{di}$ & $\mathrm{s} \times 32 \xi \times$ & $\mathrm{K}_{\mathrm{d}}=96 \mu \mathrm{s}$ & & \\
\hline \multicolumn{5}{|c|}{ Grand Total } & 315.67 \\
\hline
\end{tabular}




\begin{abstract}
ANALYSIS
Visualization

Visualization of the simulation trajectories were performed on VMD 1.9.2, the movie maker plugin was used to render the movie of self-assembly in DPC membrane. Structural analysis of the monomers were performed in Pymol 1.7 and YASARA model software suites. Images presented in the main text and in the Supporting document were all created using the above three molecular viewers.
\end{abstract}

\title{
Monomer Analysis
}

The DCD trajectory files from monomer simulation were analyzed with plugins available in VMD. RMSD was plotted using RMSD trajectory tool, and H-Bonds were computed using the hydrogen bonds plugin with donor-acceptor cut-off $3 \AA$. Heat map of individual residue RMSD was plotted using RMSD visualization tool. The equilibrium contact distance between two interacting residues shown in the main text was plotted using an in-house Tcl script.

\section{GROMACS Tools}

Tools typically found in GROMACS package were used to perform cluster analysis, weighted histogram analysis, number density profile, and diffusion co-efficient calculations. A brief description of each analysis method used in this work is provided below.

g_cluster was used to cluster dimer interfaces over the entire trajectory. Initially, individual dimers are isolated from the $10 \mu$ s snapshot after reverse mapping, and CG beads corresponding to these dimers were isolated by matching the chains. A RMSD matrix of the backbone beads of the dimers was built using the tool $g_{-} r m s$ and supplied as input for the $g_{-}$cluster tool. The last 1 $\mu$ s trajectory of the 64 monomer system was used for this analysis with frames at $20 \mathrm{~ns}$ intervals (50 frames). GROMOS ${ }^{30}$ clustering method was used with a cut off of $0.6 \mathrm{~nm}$ i.e., two structures are part of a cluster if their RMSD deviation from reference structure is below $0.6 \mathrm{~nm}$.

g_density was used to calculate the number density profiles of lipids around the protein. Only the last $200 \mathrm{~ns}$ of NPT simulation trajectory was used for these analyses at $10 \mathrm{~ns}$ timestep.

$g \_m s d$ was used to calculate the lateral diffusion co-efficient of the protein using Einstein relations.

g_wham was used to perform weighted histogram analysis. The bin size was set to 200 with -ac option to calculate integrated autocorrelation times. Error estimation was performed using Bayesian bootstrapping method. All profiles were shifted to 0 at inter protein distance of $\xi=4.0 \mathrm{~nm}$.

\section{Contact Map Analysis}


Contact maps of interacting residue chains (Figure S7) were plotted using the serves available at http://ligin.weizmann.ac.il/cma/ and https://www.molnac.unisa.it/BioTools/cocomaps/view.psp. In order to arrive at a probability distribution of interacting residues, we calculated all possible residue contacts that were within $8 \AA$ of each other from the pool of dimers observed during selfassembly simulations. The probability distribution of two residues coming in contact was calculated using the following formula

$P_{R_{a} \leftrightarrow R_{b}}=\frac{\sum x i\left(R_{a} \leftrightarrow R_{b}\right)}{\sum x i\left(R_{m} \leftrightarrow R_{n}\right)}$

where, $P_{R_{a} \leftrightarrow R_{b}}$ is the probability of residue $R_{a}$ coming into contact with residue $R_{b}$

$\sum x i\left(R_{a} \leftrightarrow R_{b}\right)$ is the total number of times residue $a$ and $b$ come in contact with each other.

$\sum X i\left(R_{m} \leftrightarrow R_{n}\right)$ is the sum of all possible residue-residue contacts observed.

The probabilities from the above calculation were normalized to the maximum value of the probability distribution

$P *_{R_{a} \leftrightarrow R_{b}}=\frac{P_{R_{a} \leftrightarrow R_{b}}}{\max \left(P_{R_{m} \leftrightarrow R_{n}}\right)}$

The calculations and probability analysis were carried out using in-house python scripts, and the plot shown in the main script is plotted using matplotlib package. 
Figure S1. Sequence alignment between Human claudin-5 and mouse claudin-15. Sequence similarity is highlighted in blue.

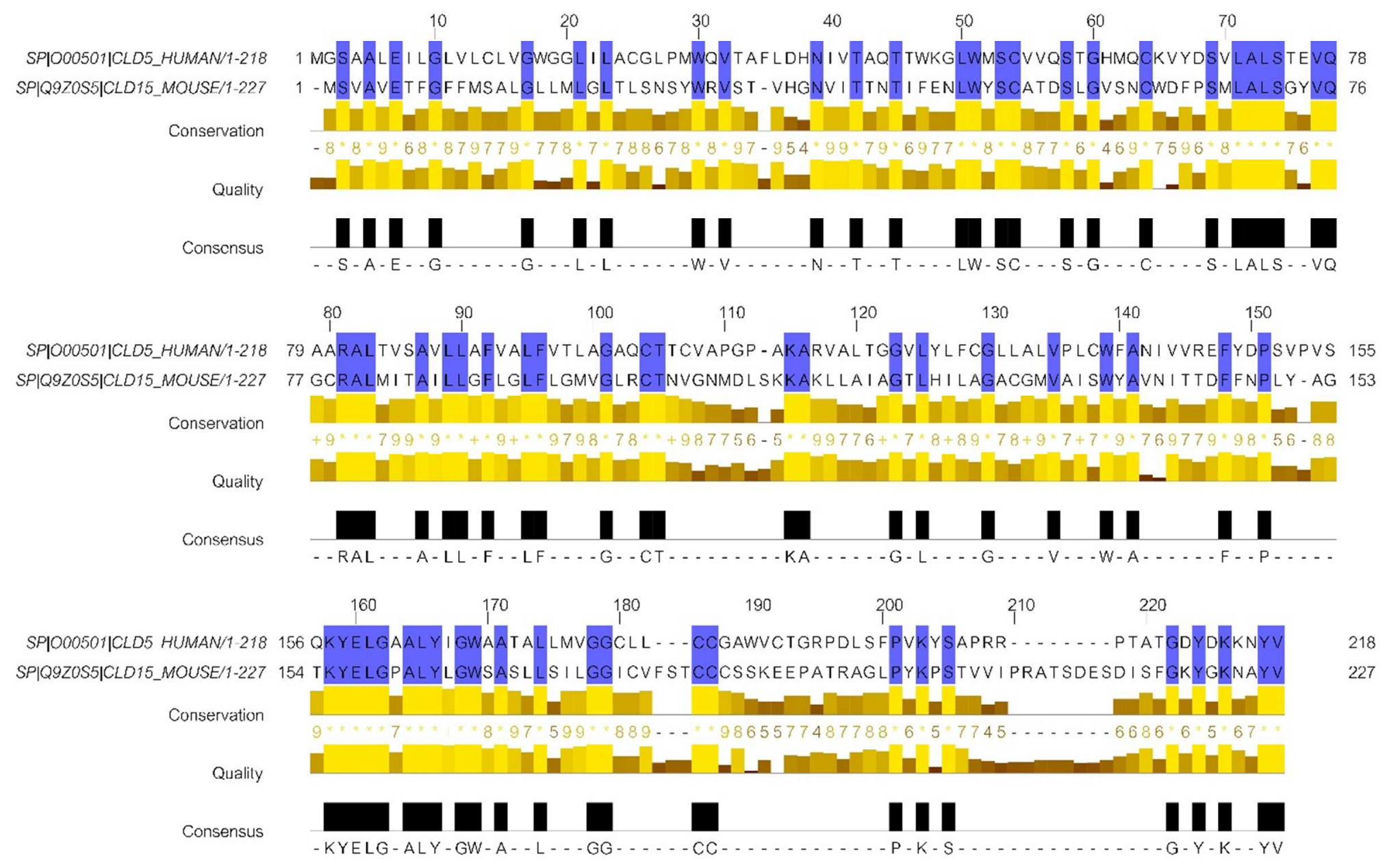


Figure S2. Multiple sequence alignment of human classic claudins (1-10 and 15) showing sequence conservation (gradient of blue) and the key residue positions (indicated with pointers shown in the legend).

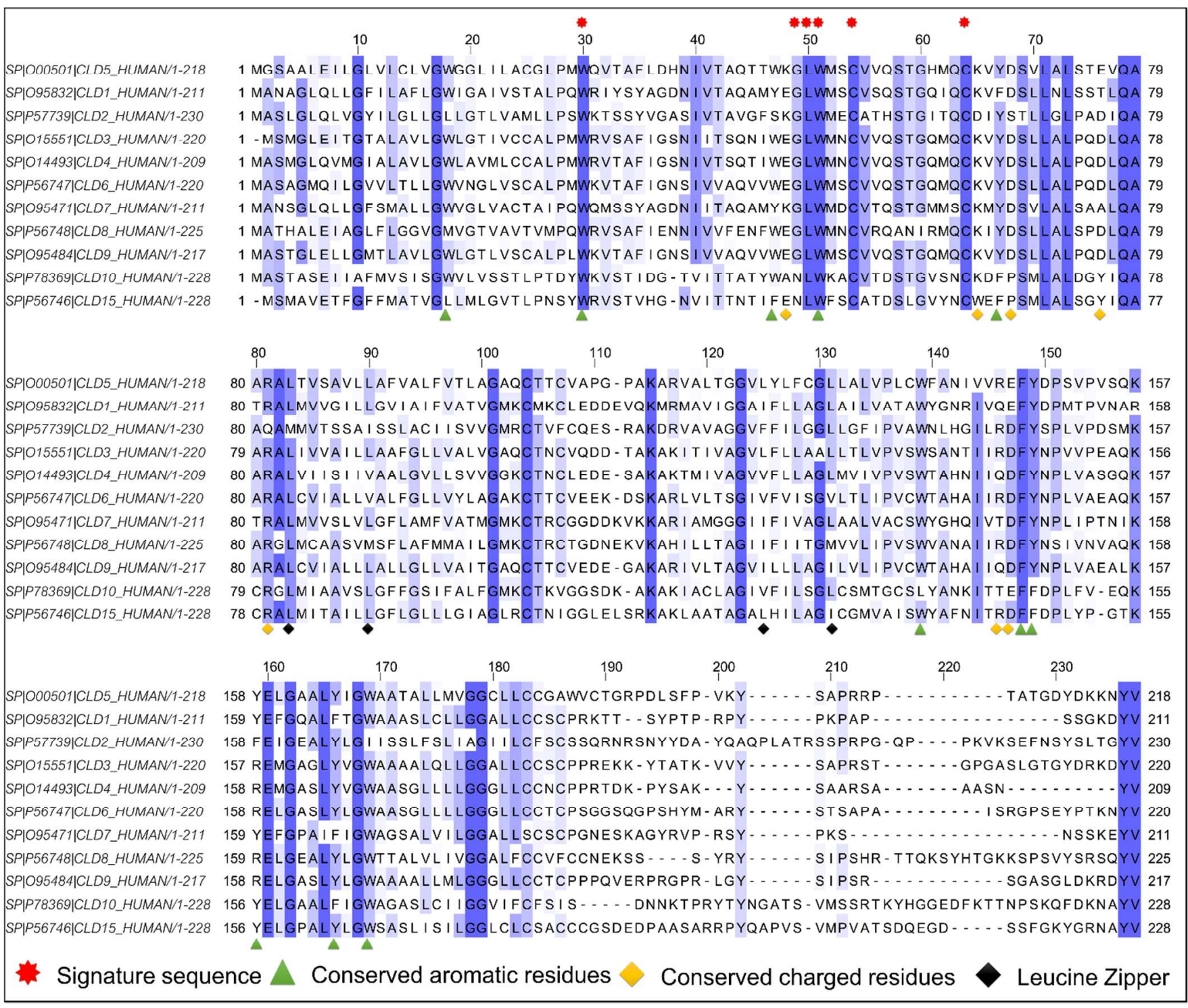


Figure S3. Structural quality measurements of the predicted claudin-5 monomer. (a) Showing Ramachandran plot of the 218 residues with Ala187 in the outlier region. (b) Showing the Z-score estimate for the structure calculated from PROSA server.

a

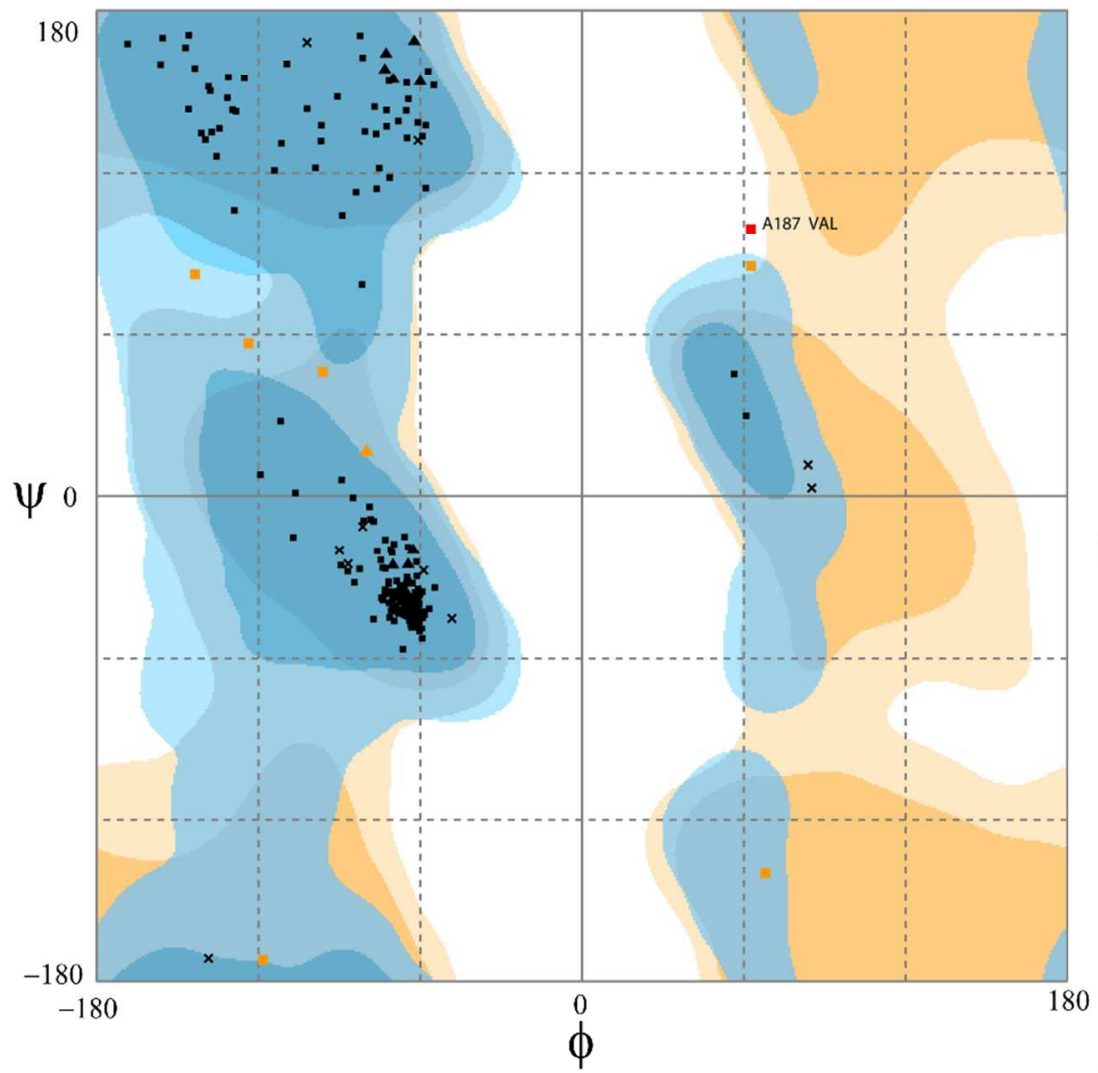

b

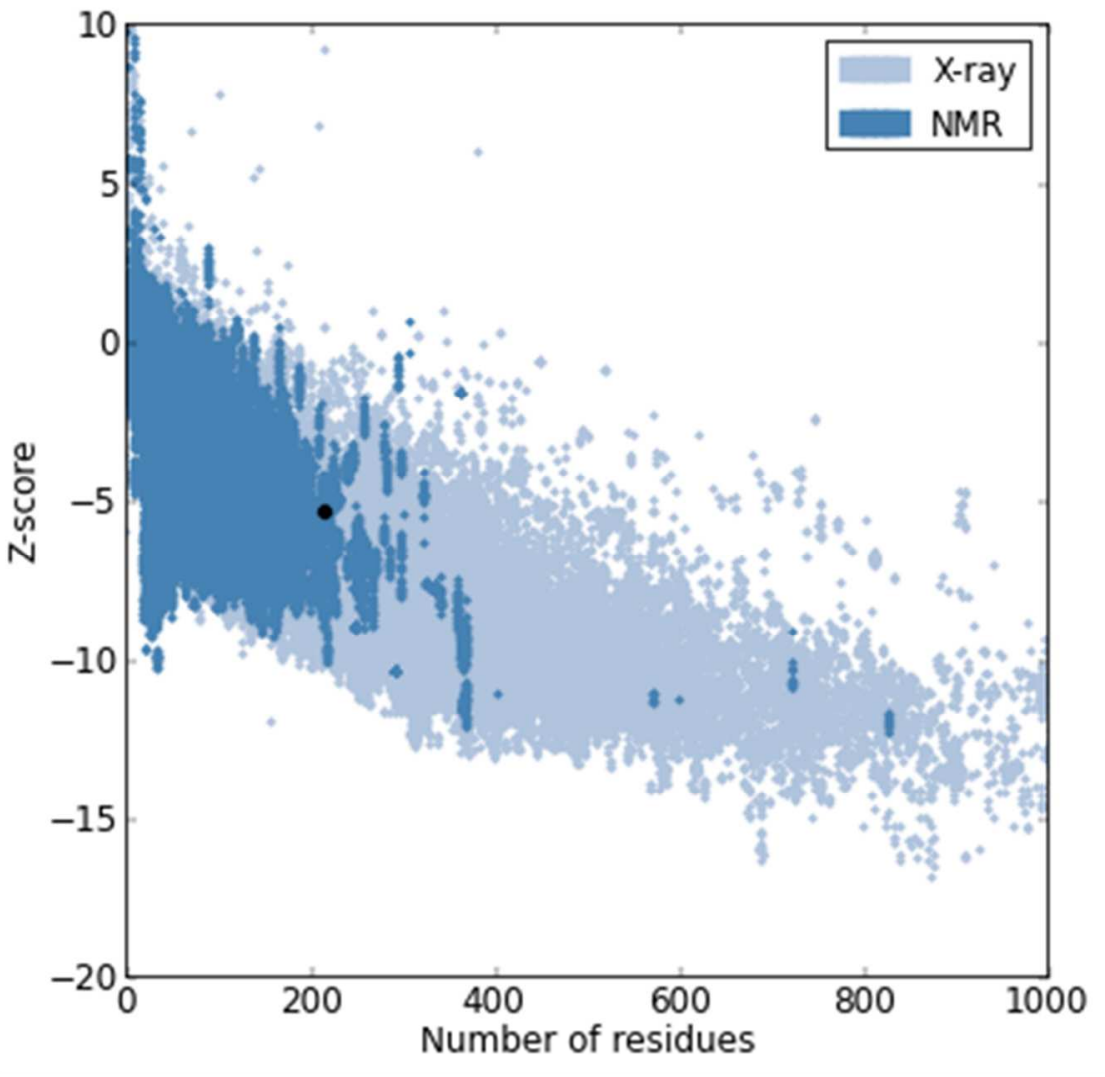


Figure S4. Topology and membrane orientation of claudin-5. (a) Showing the topology of claudin-5 in the lipid bilayer. (b) The calculated orientation of claudin-5 with respect to its hydrophobic thickness, table showing embedded residues.

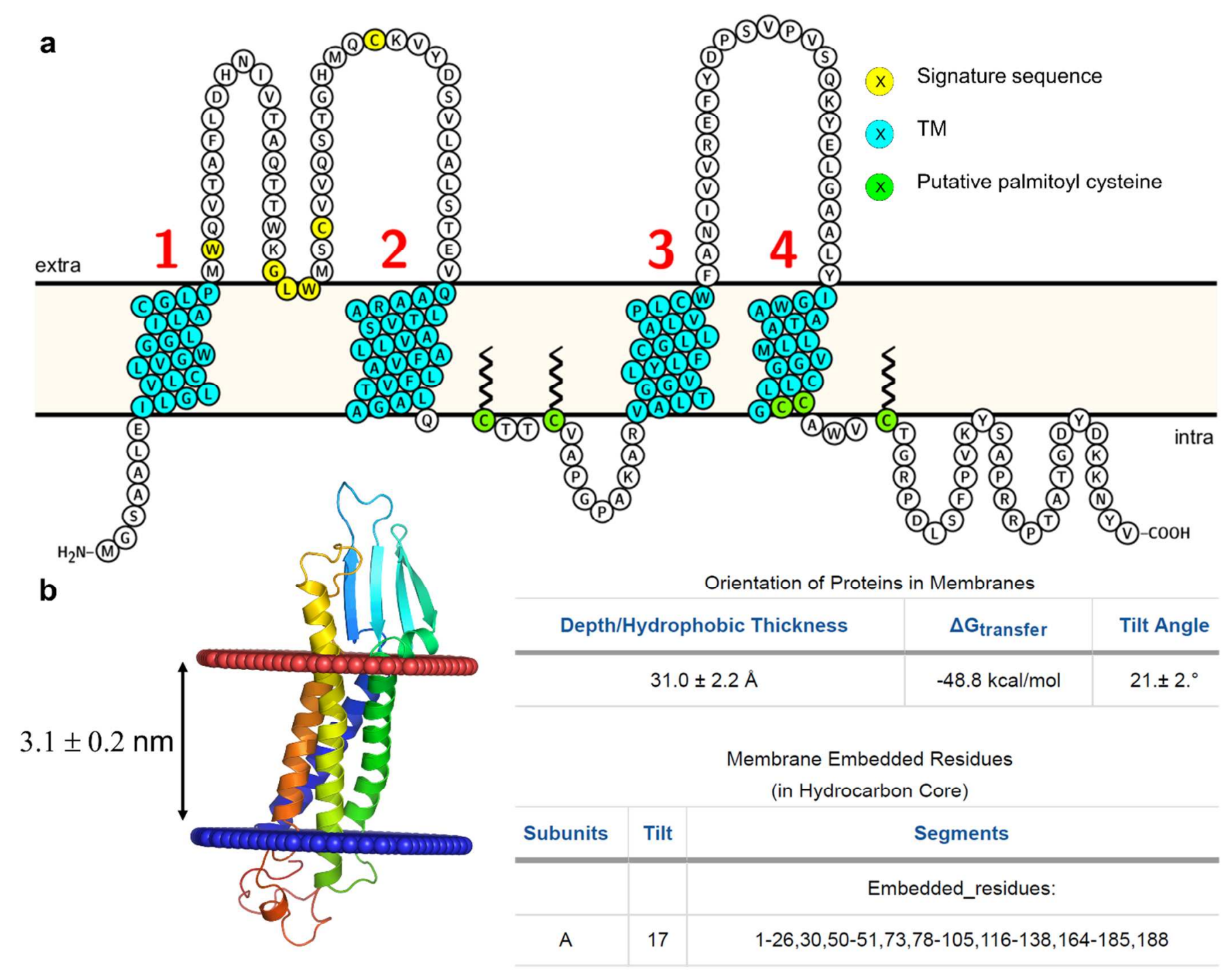


Figure S5. (a) Cutaway image of a typical $4 \times 4$ grid simulation box containing claudin- 5 (red) embedded in a lipid bilayer (cyan, green and yellow) with CG MARTINI water beads (pink) and counter ions (gray), (b) lateral view of the grid in DPC membrane, (c) number density profile of DPPC membrane around claudin-5 showing vibrational phase behavior around claudins, and (d) $10 \mu \mathrm{s}$ snapshot of the self-assembled claudin-5 monomers in DPPC bilayer.

a

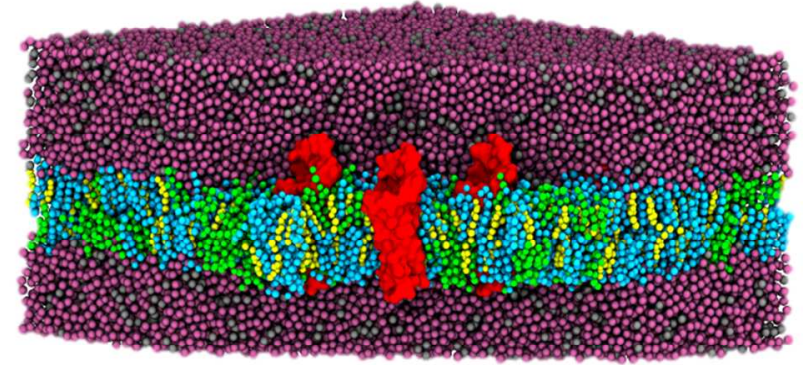

C
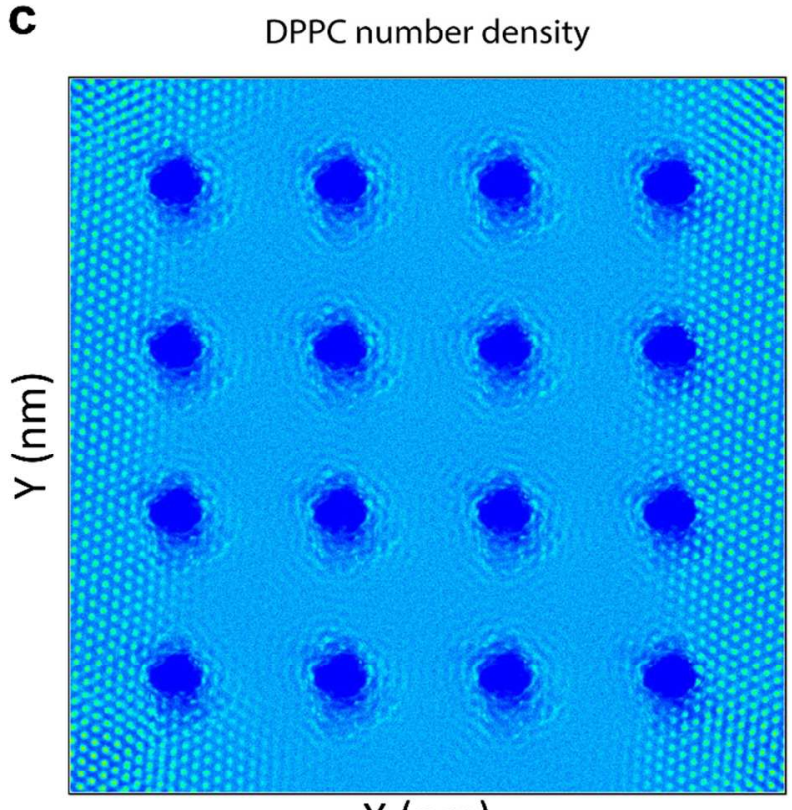

b

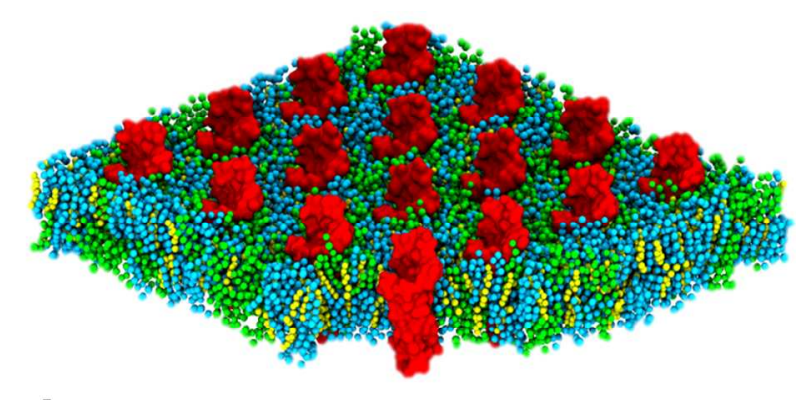

d

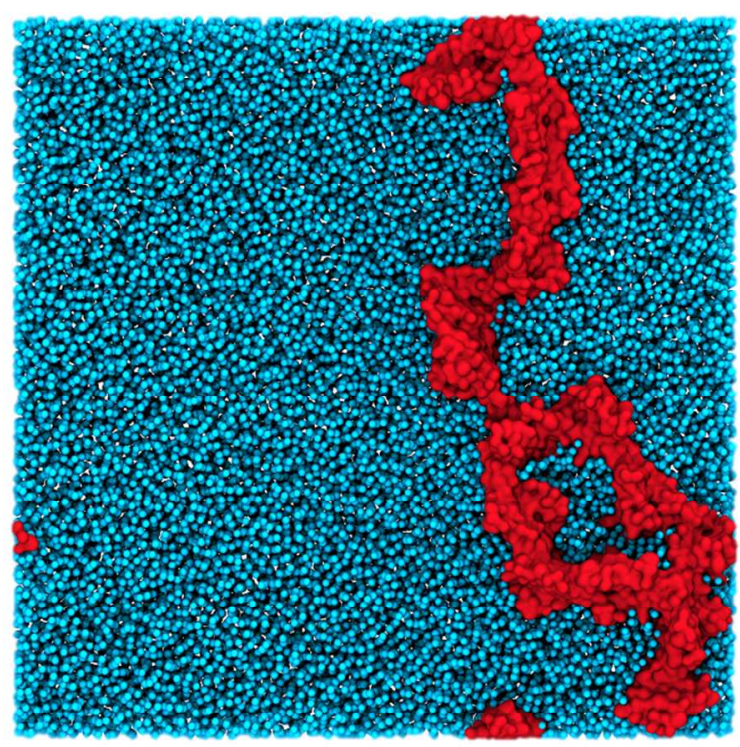


Figure S6. Top view of the $8 \times 8$ grid system (a) at $t=0 \mu \mathrm{s}$ and (b) $t=10 \mu \mathrm{s}$, (c) showing protein network without the bilayer, and (d) reverse mapped into all atom representation. The dotted circle containing a 10 monomer strand that was shown in the main text.
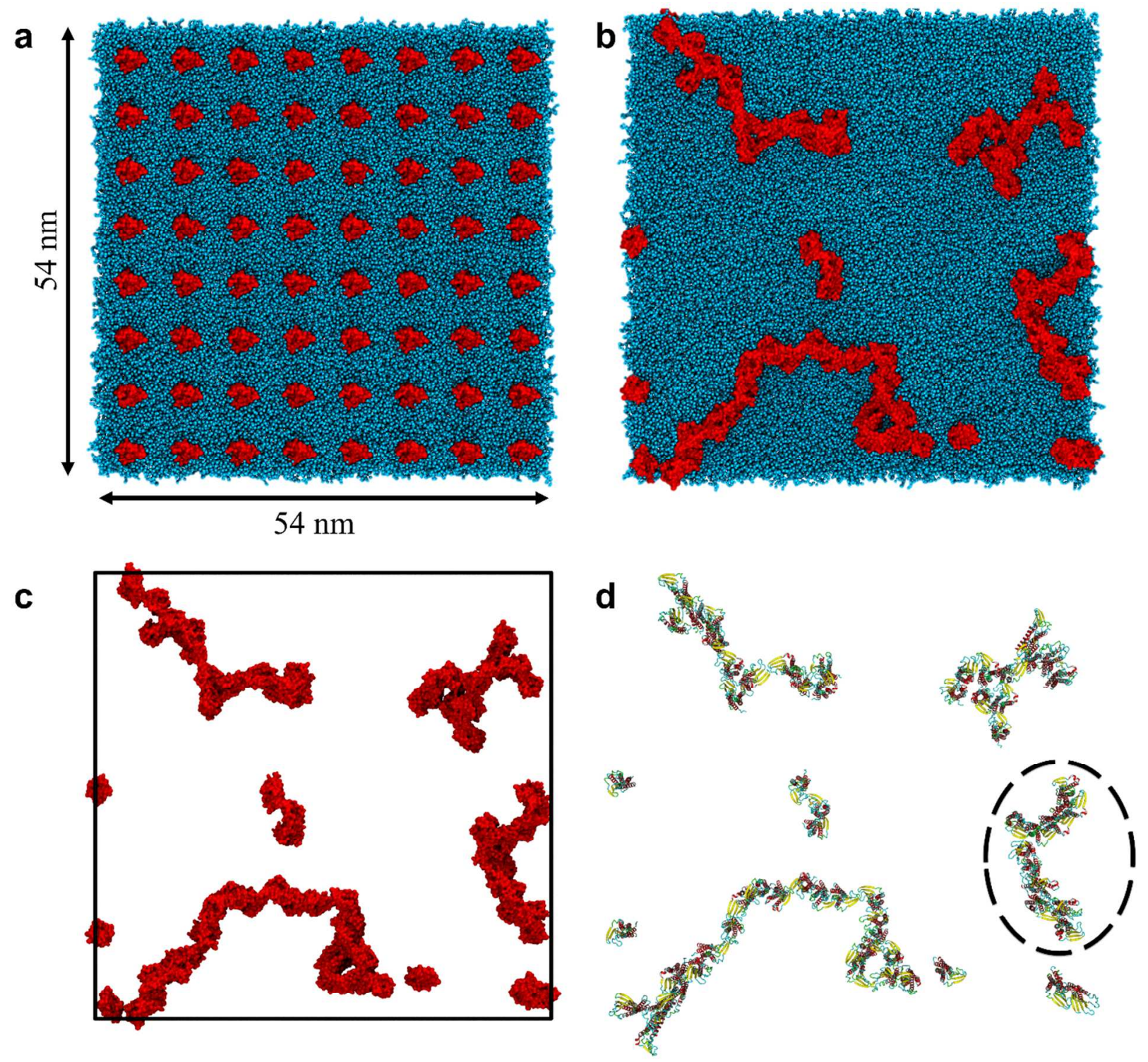
Figure S7. Formaldehyde crosslinking reaction mechanism (a) showing S1 formation of Schiffbase followed by S2 formation of a methylene bridge. Panel (b) shows dimer conformations A-D showing crosslinking interface with S1 site in red (Lys or Trp) and S2 in blue.

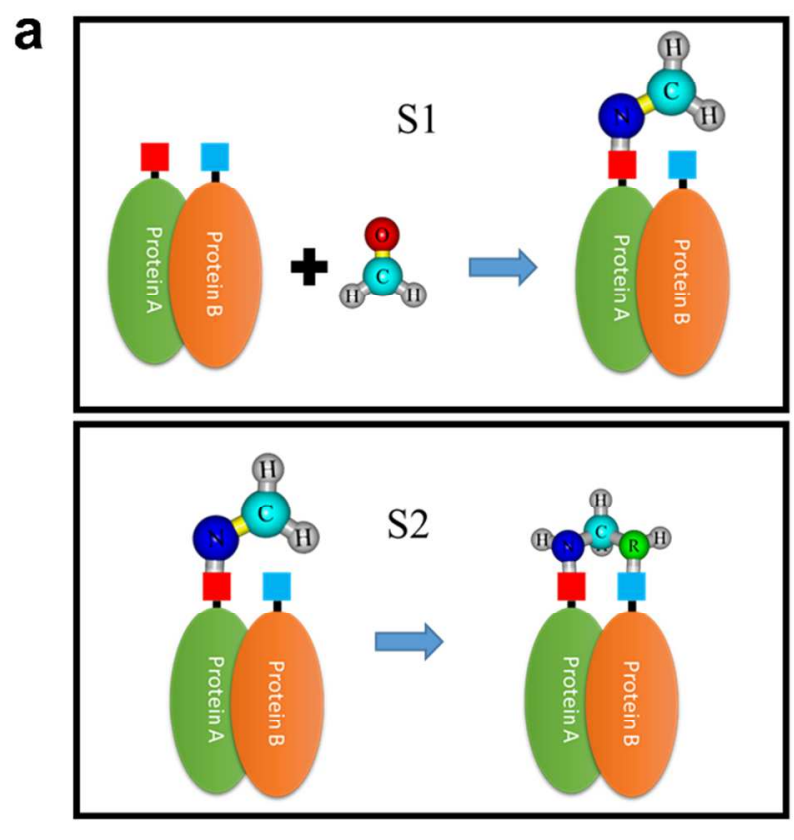

b
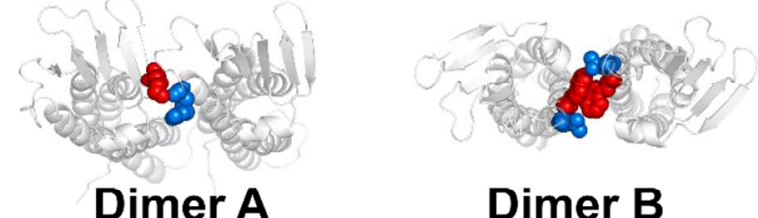

Dimer B
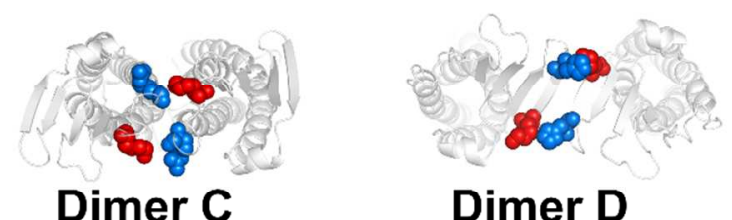

Dimer D 
Figure S8. Comparison between the claudin-15 cis interaction previously observed/predicted and claudin-5 cis interaction seen during the simulations. Panel (a) showing dimer A of claudin-5 and panel (b) shows the linear arrangement of claudin-15 observed during crystal structure (PDB:4P79) ${ }^{5}$. Panel (c) showing the ECL1 arrangement of dimer D observed in the selfassembly simulations of claudin-5 and panel (d) shows the previously published predicted model of claudin-15 ECL-1 dimer ${ }^{31}$

a

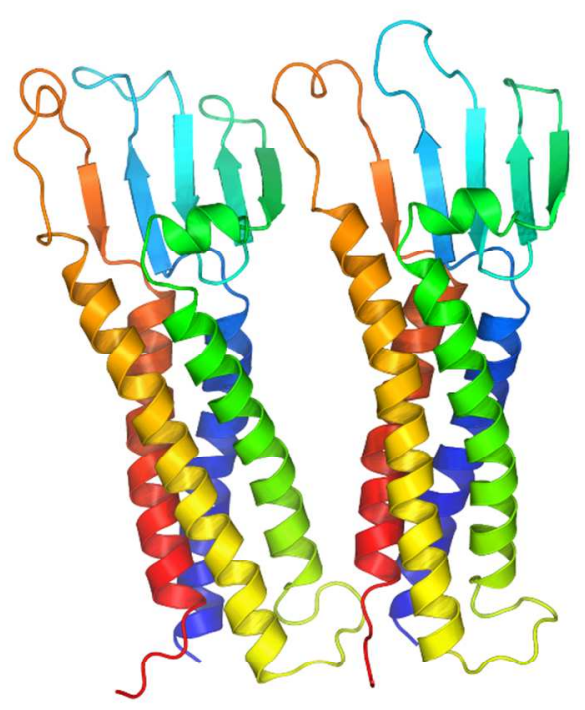

C

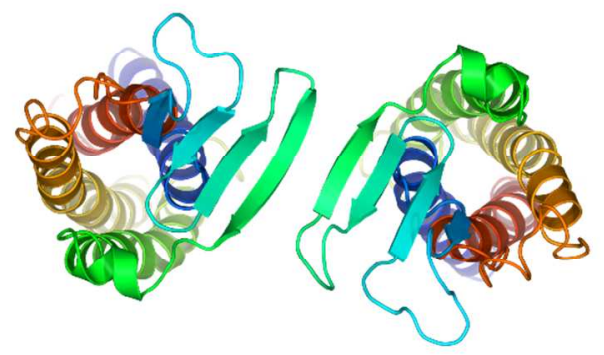

b

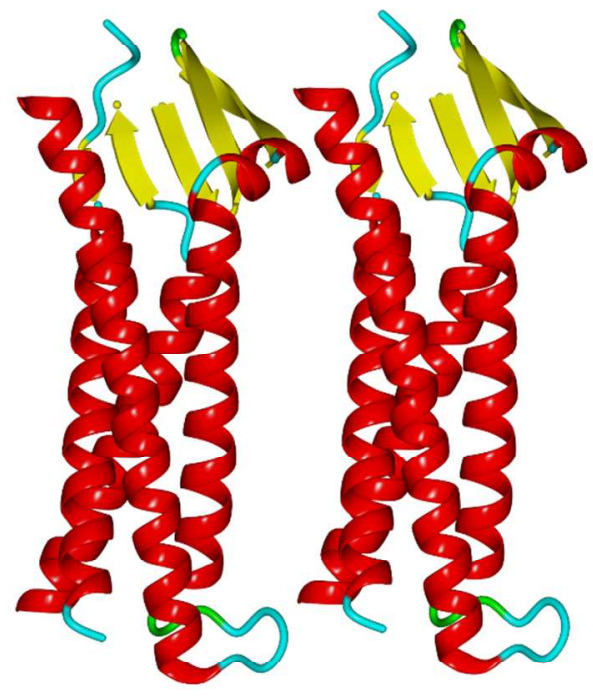

d

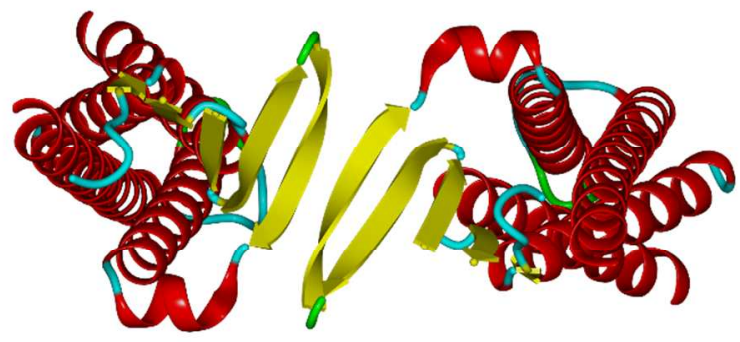


Figure S9. Contact maps of interacting residues between chains of dimers

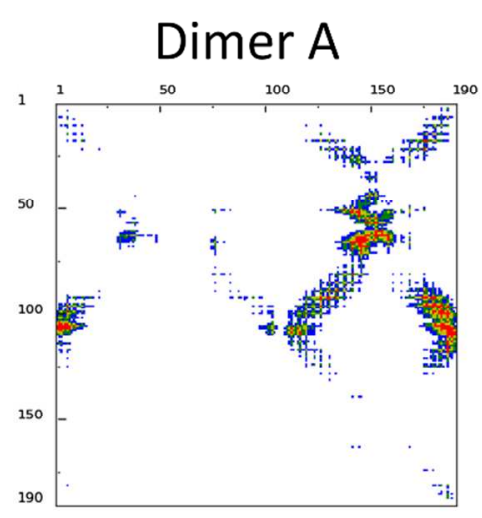

Dimer D

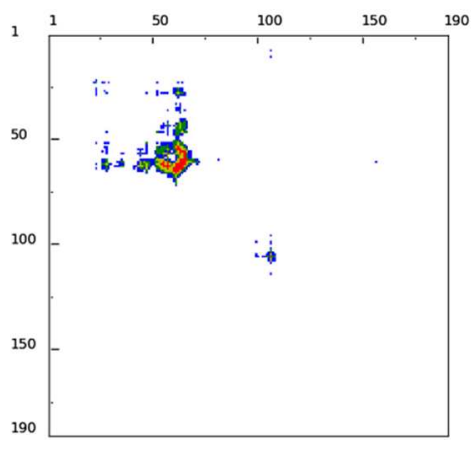

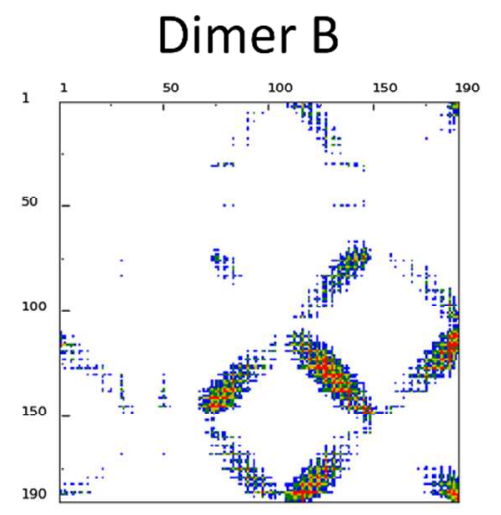

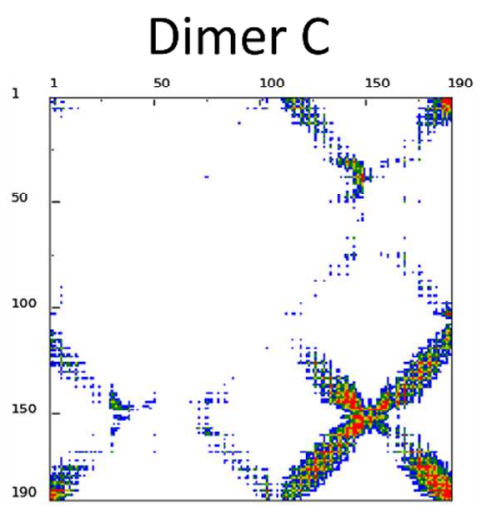

\section{Dimer $\mathrm{E}$}

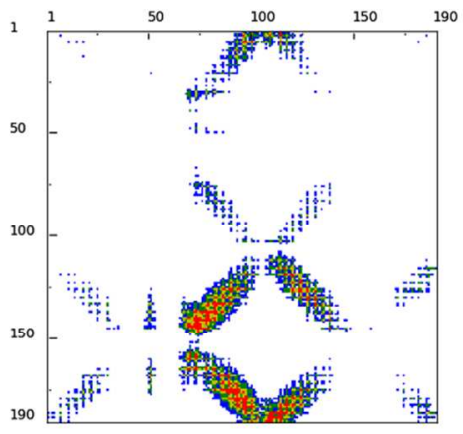

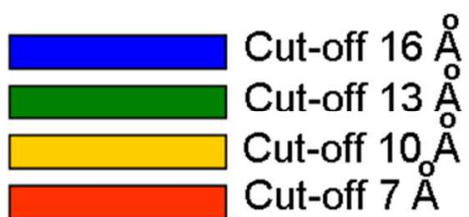


Figure S10. Steered molecular dynamics and umbrella sampling simulation set up and results. Panels show dimer B embedded in a DPPC bilayer at (a) equilibrium distance and (b) at $\xi=2$ $\mathrm{nm}$. Panel (c) shows the harmonic pull force curves the five dimers and panel (d) shows the WHAM analysis histogram with overlapping peaks corresponding to three different force constants of $750 \mathrm{~kJ} / \mathrm{mol}, 1000 \mathrm{~kJ} / \mathrm{mol}$, and $2000 \mathrm{~kJ} / \mathrm{mol}$.
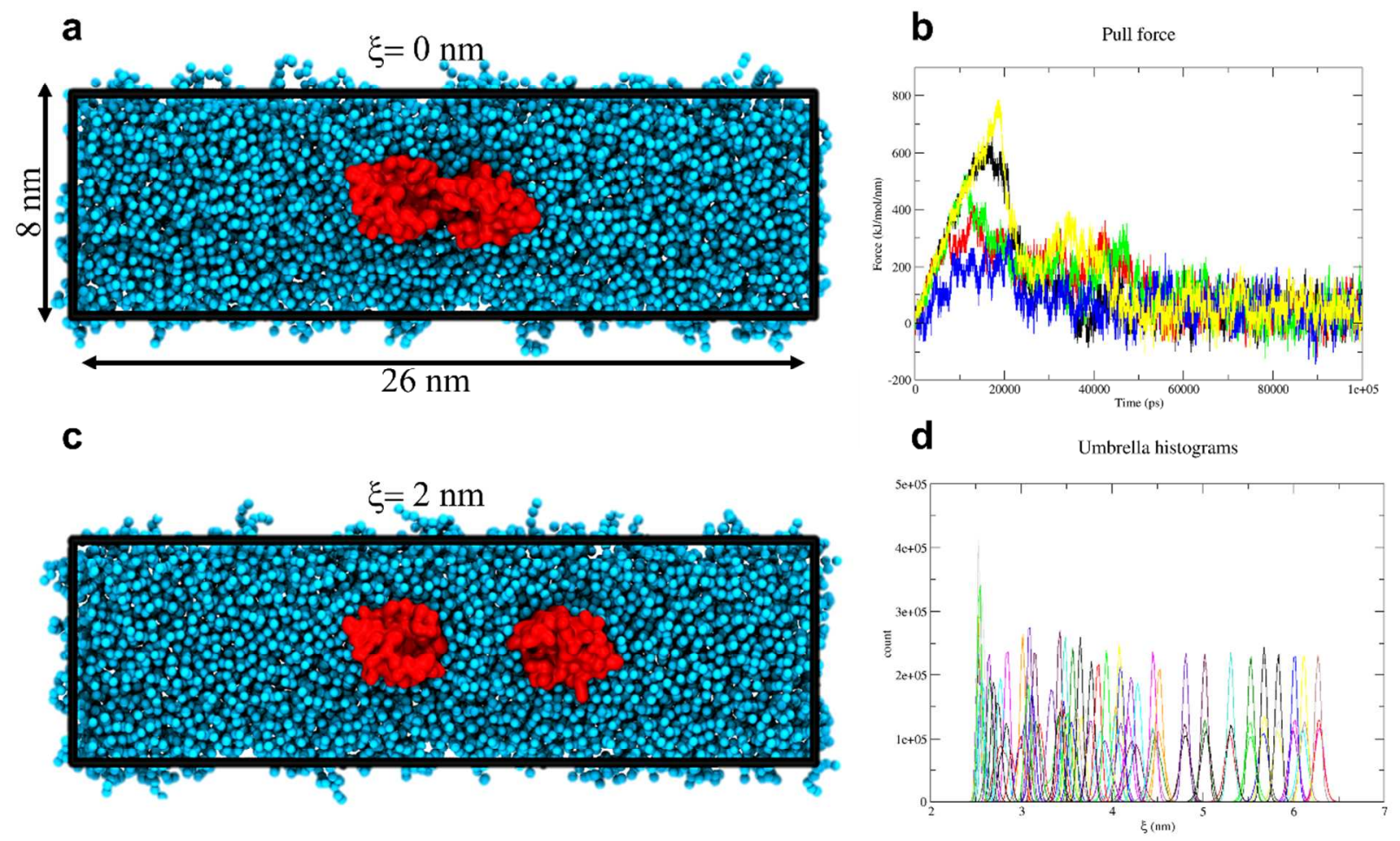
Figure S11. Structural and sequence alignment of claudin-5 and claudin-19. (a) Showing superposition of the crystal structure of murine claudin-19 PDB: 3X29 (Pink) ${ }^{32}$ with the claudin5 monomer (Cyan), the leucine residues forming the zipper domain is shown in the zoomed image. Claudin-5 residues are labeled in blues and claudin-19 residues are labeled in red. (b) Showing the sequence alignment of TM2 (80-95) and TM3 (120-135) domains from human claudin-5 and claudin-19, similarity is indicated with a ${ }^{*} *$.

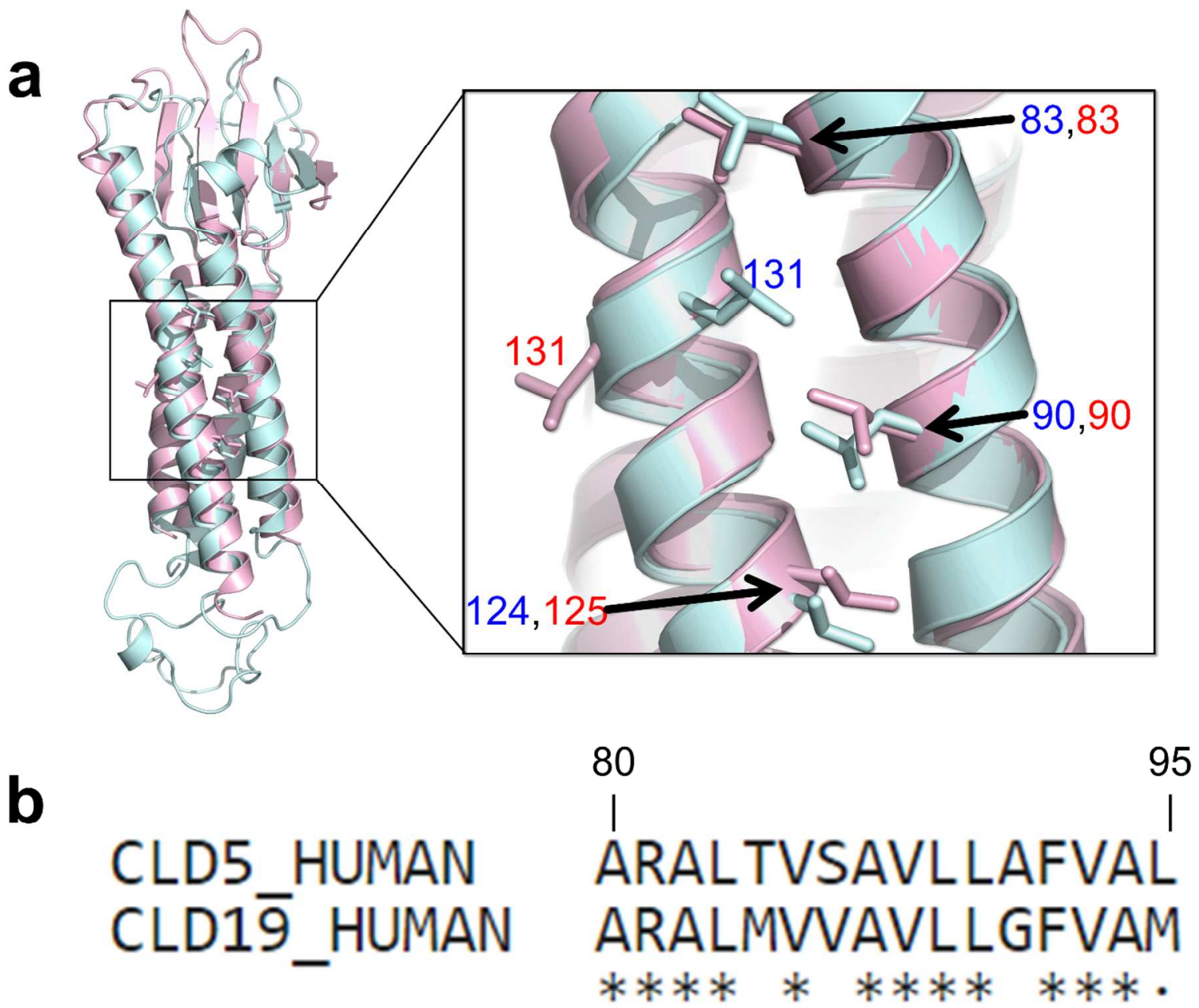

120

CLD5 HUMAN

-TGGVLYLFCGLLALVP

CLD19_HUMAN

:**.*: : : ** :*. 


\section{REFERENCES}

(1) Kelley, L. A.; Mezulis, S.; Yates, C. M.; Wass, M. N.; Sternberg, M. J. E., The Phyre2 Web Portal for Protein Modeling, Prediction and Analysis. Nat. Protocols 2015, 10 (6), 845-858.

(2) Yang, J.; Yan, R.; Roy, A.; Xu, D.; Poisson, J.; Zhang, Y., The I-Tasser Suite: Protein Structure and Function Prediction. Nat Meth 2015, 12 (1), 7-8.

(3) Biasini, M.; Bienert, S.; Waterhouse, A.; Arnold, K., et al., Swiss-Model: Modelling Protein Tertiary and Quaternary Structure Using Evolutionary Information. Nucleic Acids Research 2014, 42 (W1), W252-W258.

(4) Ebejer, J.-P.; Hill, J. R.; Kelm, S.; Shi, J.; Deane, C. M., Memoir: Template-Based Structure Prediction for Membrane Proteins. Nucleic Acids Research 2013, 41 (W1), W379-W383.

(5) Suzuki, H.; Nishizawa, T.; Tani, K.; Yamazaki, Y., et al., Crystal Structure of a Claudin Provides Insight into the Architecture of Tight Junctions. Science 2014, 344 (6181), 304-307.

(6) Xu, D.; Zhang, Y., Ab Initio Protein Structure Assembly Using Continuous Structure Fragments and Optimized Knowledge-Based Force Field. Proteins: Structure, Function, and Bioinformatics 2012, 80 (7), 1715-1735.

(7) Blaszczyk, M.; Jamroz, M.; Kmiecik, S.; Kolinski, A., Cabs-Fold: Server for the De Novo and Consensus-Based Prediction of Protein Structure. Nucleic Acids Research 2013, 41 (W1), W406-W411.

(8) Krieger, E.; Vriend, G., New Ways to Boost Molecular Dynamics Simulations. Journal of Computational Chemistry 2015, 36 (13), 996-1007.

(9) Periole, X.; Knepp, A. M.; Sakmar, T. P.; Marrink, S. J.; Huber, T., Structural Determinants of the Supramolecular Organization of G Protein-Coupled Receptors in Bilayers. J. Am. Chem. Soc. 2012, 134 (26), 10959-10965. 
(10) de Jong, D. H.; Singh, G.; Bennett, W. F. D.; Arnarez, C.; Wassenaar, T. A.; Schäfer, L. V.; Periole, X.; Tieleman, D. P.; Marrink, S. J., Improved Parameters for the Martini Coarse-Grained Protein Force Field. J. Chem. Theory Comput. 2013, 9 (1), 687-697.

(11) Lomize, M. A.; Pogozheva, I. D.; Joo, H.; Mosberg, H. I.; Lomize, A. L., Opm Database and Ppm Web Server: Resources for Positioning of Proteins in Membranes. Nucleic Acids Research 2012, 40 (D1), D370-D376.

(12) Wu, E. L.; Cheng, X.; Jo, S.; Rui, H., et al., Charmm-Gui Membrane Builder toward Realistic Biological Membrane Simulations. Journal of Computational Chemistry 2014, 35 (27), 1997-2004.

(13) Phillips, J. C.; Braun, R.; Wang, W.; Gumbart, J., et al., Scalable Molecular Dynamics with Namd. J. Comput. Chem. 2005, 26 (16), 1781-1802.

(14) Jo, S.; Kim, T.; Iyer, V. G.; Im, W., Charmm-Gui: A Web-Based Graphical User Interface for Charmm. Journal of Computational Chemistry 2008, 29 (11), 1859-1865.

(15) Klauda, J. B.; Venable, R. M.; Freites, J. A.; O’Connor, J. W.; Tobias, D. J.; MondragonRamirez, C.; Vorobyov, I.; MacKerell, A. D.; Pastor, R. W., Update of the Charmm All-Atom Additive Force Field for Lipids: Validation on Six Lipid Types. J. Phys. Chem. B 2010, 114 (23), $7830-7843$.

(16) Martyna, G. J.; Tobias, D. J.; Klein, M. L., Constant Pressure Molecular Dynamics Algorithms. The Journal of Chemical Physics 1994, 101 (5), 4177-4189.

(17) Feller, S. E.; Zhang, Y.; Pastor, R. W.; Brooks, B. R., Constant Pressure Molecular Dynamics Simulation: The Langevin Piston Method. The Journal of Chemical Physics 1995, 103 (11), 4613-4621. 
(18) Darden, T.; York, D.; Pedersen, L., Particle Mesh Ewald: An N·Log(N) Method for Ewald Sums in Large Systems. The Journal of Chemical Physics 1993, 98 (12), 10089-10092.

(19) Monticelli, L.; Kandasamy, S. K.; Periole, X.; Larson, R. G.; Tieleman, D. P.; Marrink, S.J., The Martini Coarse-Grained Force Field: Extension to Proteins. J. Chem. Theory Comput. 2008, 4 (5), 819-834.

(20) Periole, X.; Cavalli, M.; Marrink, S.-J.; Ceruso, M. A., Combining an Elastic Network with a Coarse-Grained Molecular Force Field: Structure, Dynamics, and Intermolecular Recognition. J. Chem. Theory Comput. 2009, 5 (9), 2531-2543.

(21) Wassenaar, T. A.; Ingólfsson, H. I.; Böckmann, R. A.; Tieleman, D. P.; Marrink, S. J., Computational Lipidomics with Insane: A Versatile Tool for Generating Custom Membranes for Molecular Simulations. J. Chem. Theory Comput. 2015, 11 (5), 2144-2155.

(22) Pronk, S.; Páll, S.; Schulz, R.; Larsson, P., et al., Gromacs 4.5: A High-Throughput and Highly Parallel Open Source Molecular Simulation Toolkit. Bioinformatics 2013, 29 (7), 845 854.

(23) Bussi, G.; Donadio, D.; Parrinello, M., Canonical Sampling through Velocity Rescaling. The Journal of Chemical Physics 2007, 126 (1), 014101.

(24) Nosé, S.; Klein, M. L., Constant Pressure Molecular Dynamics for Molecular Systems. Molecular Physics 1983, 50 (5), 1055-1076.

(25) Milatz, S.; Piontek, J.; Schulzke, J.-D.; Blasig, Ingolf E.; Fromm, M.; Günzel, D., Probing the Cis-Arrangement of Prototype Tight Junction Proteins Claudin-1 and Claudin-3. Biochem. J 2015, 468 (3), 449-458. 
(26) Wassenaar, T. A.; Pluhackova, K.; Böckmann, R. A.; Marrink, S. J.; Tieleman, D. P., Going Backward: A Flexible Geometric Approach to Reverse Transformation from Coarse Grained to Atomistic Models. J. Chem. Theory Comput. 2014, 10 (2), 676-690.

(27) Lemkul, J. A.; Bevan, D. R., Assessing the Stability of Alzheimer's Amyloid Protofibrils Using Molecular Dynamics. The Journal of Physical Chemistry B 2010, 114 (4), 1652-1660. (28) Kumar, S.; Rosenberg, J. M.; Bouzida, D.; Swendsen, R. H.; Kollman, P. A., The Weighted Histogram Analysis Method for Free-Energy Calculations on Biomolecules. I. The Method. J. Comput. Chem. 1992, 13 (8), 1011-1021.

(29) Roux, B., The Calculation of the Potential of Mean Force Using Computer Simulations. Comput. Phys. Commun. 1995, 91 (1-3), 275-282.

(30) Daura, X.; Gademann, K.; Jaun, B.; Seebach, D.; van Gunsteren, W. F.; Mark, A. E., Peptide Folding: When Simulation Meets Experiment. Angewandte Chemie International Edition 1999, $38(1-2), 236-240$.

(31) Suzuki, H.; Tani, K.; Tamura, A.; Tsukita, S.; Fujiyoshi, Y., Model for the Architecture of Claudin-Based Paracellular Ion Channels through Tight Junctions. J. Mol. Biol. 2015, 427 (2), 291-297.

(32) Saitoh, Y.; Suzuki, H.; Tani, K.; Nishikawa, K.; Irie, K.; Ogura, Y.; Tamura, A.; Tsukita, S.; Fujiyoshi, Y., Structural Insight into Tight Junction Disassembly by Clostridium Perfringens Enterotoxin. Science 2015, 347 (6223), 775-778. 